\title{
Corona is an Era of Tension
}

\author{
Abdallah J* \\ Chtoura hospital Bekaa, Lebanon
}

\section{Opinion}

Anxiety is one of the most common mental health problems in the world, which has led to drastic changes in humans that have occurred in all aspects of our lives during the past months, to an increase in anxiety levels, especially among parents, who feel increasingly concerned about the health of their children and relatives, so the feeling of anxiety More than just a feeling of tension or anxiety, there are natural reactions that we all feel at some point in our life and it may be a new tension or anxiety other than what we used to do before the Corona, but constant anxiety appears as if it is permanent fear and if the situation worsens, it may control your life and prevent you from doing business The normal daily routine. Tension and deep fatigue make you feel anxious, tired and inability to concentrate at all times. This may disrupt sleep and daily life and make you feel depressed.

And often there are symptoms of tension on the body as well, such as a rapid heartbeat or difficulty breathing, a feeling of trembling, sweating, dizziness, diarrhea, and nausea, and these symptoms may continue psychologically and physically with the patient who was infected with Corona and sometimes it affects the person who delusions Corona disease and did not suffer from Corona so suffers 2/10 from People who have been exposed to corona from a problem of anxiety or phobia and at some point in their lives, but it is necessary for many of them to obtain treatment or psychological treatment.

We know that the outbreak of the Corona disease imposed on people around the world to stay in their homes, closed borders, and world economies shook under the weight of the spread of virus infection, and it may be difficult for one to see all these events unfold in front of him without being grieving and suffering from depression, but there are several proactive steps that may be We do it to improve your mood even during times of worries and sorrows.

There is a strong relationship between the increase in psychological problems and the spread of the Corona epidemic that causes anxiety and may reach panic states that afflict people with corona and the accompanying symptoms and psychological disorders. Many of those who suffer from the Corona epidemic are exposed to severe psychological crises and stress on their family and work lives, and may lead to severe symptoms and it could lead to death. The social isolation that was approved and advised by doctors and global health organizations increased exposure to psychological disorders and severe sadness during confinement of the person himself in one room for a period of three weeks, so that measuring the rate of anxiety and depression made many of those who were infected with Corona have problems with memory and sleep disorders and it is worth It is noteworthy that many people who have mental illnesses are more likely to suffer from the Corona epidemic and have many serious and permanent complications at a higher rate than ordinary people.

Therefore, I offer daily practical advice on how to deal with psychological pressure in a better way,

For example, these are my daily revelations, stop thinking about Corona.When a topic worries you and raises your fears, whether it is the outbreak of the new Corona virus or financial crises or otherwise, you may find yourself against your will reading the news and following the development of crises all the time, but recalling these stressful events over and over again is related Blood pressure rises and mood changes. Distraction and stopping thinking may contribute to lower stress triggers and lower daily panic rates.

First: Stop thinking about Corona by all means and from all media, press and other means.

Second: Not helping or encouraging him to practice suggestion because it may increase anxiety and tension for some and may take it as an opportunity to ruminate negative thoughts and increase psychological pressure, so instead of clearing their minds, they may distract them with necessary things.

Third: Exercise is a very important thing, such as walking in the fresh air to get an amount of oxygen. Or, for example, practicing yoga or pushing against the wall, among others.

Fourth: Social distancing is also a cause of psychological delusion

\begin{tabular}{|c|l|}
\hline \hline Quick Response Code: & *Corresponding author: Jarrah Abdallah, Chtoura hospital Bekaa, Bekaa, Lebanon \\
Received: 07 May, $2021 \quad$ Published: 13 May, 2021 \\
Citation: Abdallah J. Corona is an Era of Tension. J Psych Sci Res. 2021;1(2):1-2. D0I: \\
$10.53902 / J P S S R .2021 .01 .000506$
\end{tabular}


Fifth: The news of the spread of Coronavirus has caused many people to increase anxiety and tension around the world, so we have to get rid of these negative feelings in the right ways.

Sixth: There are some actions that can be done at home that may help relieve stress, such as practicing their favorite activities such as: reading, watching comedies, or talking with friends and having a good sleep.

\section{Acknowledgments}

None.

\section{Funding}

None.

\section{Conflicts of Interest}

Author declares that there is no conflict of interest. 